\title{
An evaluation of the completeness of reporting of childhood tuberculosis
}

\author{
S.S.S. Teo*, M. Alfaham ${ }^{\#}$, M.R. Evans ${ }^{\Uparrow}$, J.M. Watson ${ }^{+}$, A. Riordan ${ }^{\S}$, P. Sonnenberg ${ }^{f}$, \\ J. Clark**, A. Hayward ${ }^{\# \#, ~ M . ~ S h a r l a n d ~}{ }^{4 \uparrow}$, J. Moore-Gillon ${ }^{++}$, V. Novelli ${ }^{\S \S}$, D. Quinn ${ }^{+}$, \\ D. Shingadia ${ }^{\S \S}$ for the British Paediatric Surveillance Unit Childhood Tuberculosis \\ study group
}

ABSTRACT: The sensitivity of the Enhanced Tuberculosis Surveillance (ETS) scheme for monitoring tuberculosis in children is unknown.

We used the British Paediatric Surveillance Unit (BPSU) reporting scheme to conduct a prospective observational study of tuberculosis in children aged $<16$ yrs in the UK. Reported cases were then matched with records from the ETS database.

A total of 320 cases were reported to the BPSU between January and December 2004. We estimated that there were 557 paediatric cases in England, Wales and Northern Ireland in 2004: $222(40 \%)$ cases reported to both BPSU and ETS, 98 (18\%) reported to BPSU but not ETS and 237 (42\%) reported to ETS but not BPSU. Children aged $<5$ yrs were significantly less likely to be reported to ETS compared with older children $(p<0.01)$.

There is substantial under-reporting of childhood tuberculosis, especially of children aged $<5 \mathrm{yrs}$. ETS provides a representative picture of the demographics but may miss $\sim 20 \%$ of cases. This should be taken into account when planning training and resource requirements for tuberculosis. Increased efforts are needed to ensure that all paediatric cases are reported to ETS.

KEYWORDS: Child, disease notification, medical record linkage, population surveillance, tuberculosis

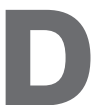
ata on tuberculosis (TB) in children in the UK and Republic of Ireland (ROI) are collected by several separate surveillance systems. The Enhanced Tuberculosis Surveillance (ETS) system and the statutory Notifications of Infectious Diseases System cover England, Wales and Northern Ireland, although statutory notifications provide only limited demographic and clinical data [1]. Mycobnet is a laboratory-based surveillance system for Mycobacterium tuberculosis complex isolates and their antibiotic susceptibilities, that includes Scotland [2]. There are also separate TB surveillance systems in Scotland and the ROI run by Health Protection Scotland and the Irish Health Protection Surveillance Centre, respectively. Although TB is a statutorily notifiable disease in all these countries, under- and over- notification are recognised to occur $[3,4]$. However the extent of under- or over-notification at a national level is unclear, especially for children.

Effective surveillance should be representative of all cases in the population. In the UK, TB services generally involve adult chest physicians and TB nurses in designated TB clinics. Paediatricians have traditionally not been part of these clinics and have only recently developed closer working links with TB services. It is possible that cases seen by paediatricians who do not work within TB services might be systematically excluded from established surveillance systems, particularly in low-incidence areas. Reliable demographic information is important for policy formulation in the control and prevention of TB; however, the extent of the potential exclusion of childhood TB cases from national surveillance systems, such as the ETS, has not been fully evaluated.

Our aim was to determine the extent of underreporting of childhood TB in the UK by matching cases reported to a specially commissioned paediatric surveillance system with records from the routine ETS.

\section{METHODS}

\section{Study design}

Cases were prospectively identified between December 2003 and January 2005 by the British Paediatric Surveillance Unit (BPSU) of the Royal College of Paediatrics and Child Health using

\section{AFFILIATIONS}

${ }^{*}$ Centre for Child Health, University of London,

${ }^{+}$Respiratory Diseases Dept, Health Protection Agency, Centre for Infections,

${ }^{f}$ Centre for Sexual Health and HIV Research,

${ }^{\# \#}$ Centre for Infectious Disease Epidemiology, University College London,

${ }^{++}$Dept of Respiratory Medicine, St Bartholomew's Hospital and Royal London Hospital,

${ }^{\S \S}$ Dept of Infectious Diseases, Great Ormond Street Hospital,

"Paediatric Infectious Diseases

Unit, St George's Hospital, London,

"Dept of Child Health, Llandough

Hospital,

'Communicable Disease

Surveillance Centre, National Public

Health Service for Wales, Cardiff,

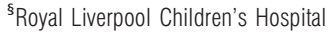

(Alder Hey), Liverpool, and

${ }^{* *}$ Dept of Paediatrics, Newcastle

General Hospital, Newcastle, UK.

\section{CORRESPONDENCE}

D. Shingadia

Dept of Infectious Diseases

Great Ormond Street Hospital

Great Ormond Street

London WC1N 3JH

United Kingdom

E-mail: shingd@gosh.nhs.uk

Received:

Feb 292008

Accepted after revision:

Feb 042009

First published online:

Feb 272009

European Respiratory Journal

Print ISSN 0903-1936

Online ISSN 1399-3003 
methods previously described [5]. These depend primarily on case reports by consultant paediatricians, since other health professionals involved in TB services, such as chest physicians and TB nurses, are not part of the BPSU scheme. Consultant paediatricians in the UK and ROI are sent monthly reporting cards. The overall 2004 response rate for all BPSU studies was $91.2 \%$ [5]. When a case was reported to the BPSU, the reporting clinician was sent a postal questionnaire requesting further details of the case. Nonrespondents were prompted by repeat questionnaires and by telephone contact. Respondents were contacted for clarification if any data were missing from the returned questionnaire. In order to ensure confidentiality, personal identifier data on cases were restricted to year of birth, sex and partial postcode. This study was approved by the South and West Multi-Centre Research Ethics Committee in the UK.

\section{Case definitions}

Cases were defined as children $<16$ yrs of age in the UK and ROI with newly diagnosed TB who started treatment in 2004. Cases were categorised as either: confirmed cases, i.e. cultureconfirmed disease due to Mycobacterium tuberculosis complex infections (M. tuberculosis, M. bovis, M. africanum); or probable cases, i.e. not culture confirmed but with a clinical or radiological diagnosis of $\mathrm{TB}$ and treated with two or more anti-tuberculous drugs.

\section{Statistical analysis}

Cases were mapped where possible to country and, within England, to London only and England excluding London using the National Health Service (NHS) postcode directory. Statistical analysis was performed using Stata 9.0 (StataCorp, College Station, TX, USA). Fisher's exact test was used to compare categorical variables.

\section{Matching of the study and ETS datasets}

Reports from the BPSU from England, Wales and Northern Ireland were matched with records from the ETS database (from October 2003 to March 2005). The longer period for the ETS database was chosen as reports are dated by the date the report was completed. In contrast, BPSU reports are dated by the date that treatment was started. Cases from Scotland and ROI were excluded as ETS does not cover these countries. The matching criteria were year of birth, sex, partial postcode, ethnicity, place of birth (UK, ROI or abroad) and the date treatment was started. For children born abroad, the variables country of birth and year of entry to the UK were also used. We describe matched and unmatched records from the BPSU dataset and describe unmatched records from the ETS dataset over the calendar year 2004 .

An in-house algorithm was designed in Microsoft Office Access (Microsoft Corp., Redmond, WA, USA) that automatically matched pairs of records based on the above criteria. The algorithm automatically ranked pairs of records by score, with a higher score representing a higher likelihood of a true match. Pairs of records were classified as a match if data matched exactly on all fields. Pairs of records were also classified as a match if the year of birth, sex and partial postcode all matched, even where data for ethnicity, country of birth and the year of entry were missing. The algorithm automatically classified remaining pairs of records as mismatches or possible matches.

Subsequent human review enabled reclassification of possible matches as matched pairs or mismatches. This review was based on similarities in the aforementioned fields, the date of notification (as opposed to the date that treatment was started) and the notifying centre. A threshold of 10 days' difference in the entry of the date that treatment had been started was accepted as sufficient to classify this field as matched. Finally, we manually verified automated matches and performed deduplication of matched pairs to remove the possibility of double-counting.

\section{RESULTS}

\section{Reporting}

Over the 14-month surveillance period, there were 566 reports to the BPSU through the monthly reporting system. Cases were excluded if no other information was received from the reporting paediatrician $(n=40)$, or if reports were duplicates $(n=59)$, fell outside the surveillance period $(n=31)$, were cases that only received chemoprophylaxis $(n=12)$ or were due to nontuberculous disease such as nonmycobacterial diseases $(n=12)$, nontuberculous mycobacterial infections $(n=11)$ or Bacillus Calmette-Guérin (BCG) reactions $(n=5)$. Six cases were outside the age range and five were erroneously reported for other reasons. In addition, we excluded 65 cases that were either from Scotland or ROI or were diagnosed in December 2003 or January 2005, leaving 320 eligible cases for the matching process.

\section{Matching}

From the BPSU dataset 320 records from England, Wales and Northern Ireland were compared to 446 records from the ETS dataset. In total, $222(69 \%)$ of BPSU records were matched to an ETS record. The overall number of cases was 557 divided as follows: $222(40 \%)$ in both BPSU and ETS databases, 98 (18\%) reported only to BPSU and $237(42 \%)$ reported only to ETS (table 1).

Children aged $\leqslant 4$ yrs were less likely to be matched than those aged 5-9 yrs $(\mathrm{p}=0.01)$ and $10-14$ yrs $(\mathrm{p}<0.001)$ but as likely as those aged 15 yrs children to be matched $(p=0.11)$. A higher proportion of cases in London (83\%) were matched compared with cases in England but outside London $(62 \%$; $\mathrm{p}<0.001)$, while children who were culture positive $(80 \%)$ were more likely to be matched than those who were culture negative $(61 \% ; \mathrm{p}=0.003)$.

\section{DISCUSSION}

This project was an active surveillance system specially set up with paediatricians, in part, specifically to estimate undernotification in the routine ETS system. We estimated that there were 557 paediatric TB cases in England, Wales and Northern Ireland in 2004: 40\% reported to both the BPSU study and ETS, $18 \%$ reported to the BPSU only and $42 \%$ reported to ETS only. The 98 records in our dataset not matched to records in ETS suggest that the burden of TB in children in England, Wales and Northern Ireland, at least, may be underestimated by about one-fifth, particularly in children aged $<5$ yrs. Undernotification of TB is a recognised problem in both adults and 


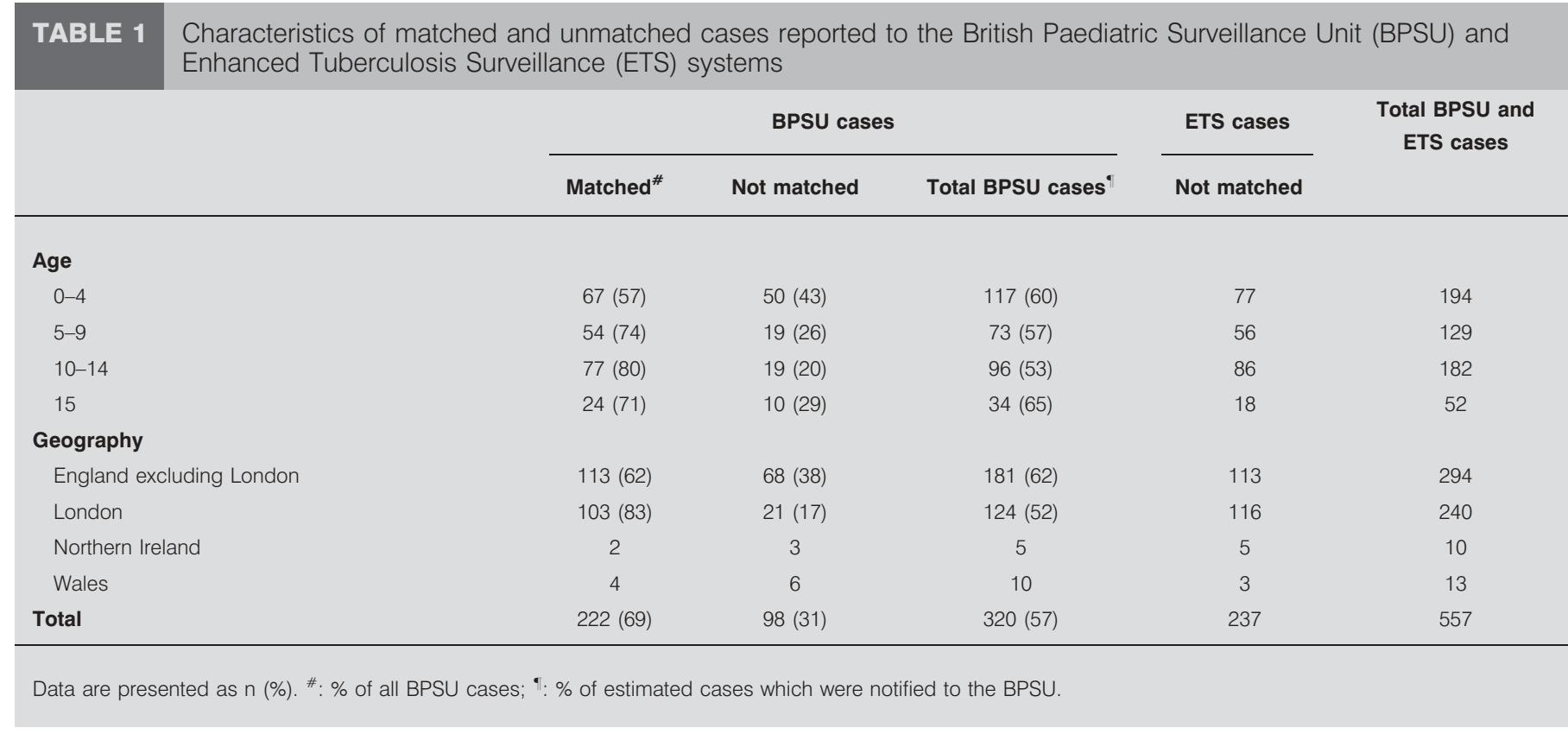

children but the completeness of reporting to the ETS scheme has been unknown $[3,6]$.

Our estimates will be subject to two sources of measurement error. Probable missed matches would lead to an overestimation of the number of cases. In addition, the cases that were not caught in either source and that have been excluded from the matching process would tend to underestimate the number of cases. However in the UK National Health Service, all children have access to specialist paediatric services so that our results are unlikely to be explained by poor access to, or diagnosis without, the state health care system.

ETS commenced in 1999 [1] but only London-based TB services currently report electronically. With the specific aim of examining TB cases aged $<16$ yrs only, we also hoped to validate and determine the representativeness of ETS at the national level. We estimated, however, that even with these restrictions, matching only on year of birth, sex and partial postcode would result in a $10 \%$ false positive matching rate, i.e. $\sim 40$ records. We suggest that by using additional fields we were able to decrease this false positive rate to $1 \%$. While our probabilistic approach could not be applied to the pairs of records identified by our algorithm that had contradictory data, we suggest that our matching process is likely to have underestimated the number of matches due to data quality and incompleteness of data.

The matching algorithm did not require an exact match on all fields to automatically match pairs of records, while subsequent human review allowed further flexibility to decrease the number of false negative matches. In addition cases reported to ETS may have been reported to BPSU but if no other data were sent, these would have been excluded from the matching process.

Capture-recapture methodology estimates the number of cases that may have been missed by two or more reporting systems, allowing an estimate of the total number of cases in a population. However this form of analysis requires certain assumptions, which were not met in our study population. Reporting to the two systems may not have been independent [7]; paediatric services with large caseloads may have preferentially reported to ETS rather than to the BPSU. In addition, the probability of each case being identified may not have been homogeneous [7, 8]. We also suspect that adult physicians may be more likely to manage older children with TB. These older children will not have been reported to the BPSU but may have been reported to ETS.

The possible impact of the BPSU study on reporting rates to routine public health surveillance was a concern during the planning stages. We therefore encouraged paediatricians to report to both. However it is difficult to estimate what impact the BPSU study may have had on reporting rates to other systems. The number of reports of children aged $\leqslant 15 \mathrm{yrs}$ to ETS was 452 and 389 in 2002 and 2003, respectively. In 2004, there were 446 reports, suggesting that there was no adverse effect on completeness of reporting of paediatric cases to ETS.

However, the large number of children reported to ETS but not to our study suggests that either paediatricians did not report them to the BPSU, or that these cases were not seen by a paediatrician. If the latter is correct this may imply that approximately two-fifths of the children with TB in these countries have not had a paediatrician involved in their management. Both the British Thoracic Society and National Institute for Health and Clinical Excellence guidelines state that children should be managed either by a paediatrician with special experience and training in $\mathrm{TB}$ or by a general paediatrician working with a physician appropriately trained in ТB $[9,10]$. Furthermore, the children's National Service Framework promotes integrated clinical networks, including specialist clinical networks and local providers of paediatric services [11]. 
Children seen by paediatricians in London were more likely to be reported to both systems than those in the rest of England. This difference may be due to the availability of web-based reporting via the London TB Register [12]. Even so, there were 116 children in London notified to ETS but not matched to a BPSU case. Perhaps children seen in paediatric TB clinics (with access to the web-based reporting) were under-reported to our study due to TB workload issues, or these children were not seen by paediatric services. We suspect a combination of both is the cause. Culture-positive children were also more likely to be reported to both systems presumably because they had a more definitive diagnosis of TB.

The youngest children (aged $\leqslant 4$ yrs) were less likely to be reported to ETS than those aged 5-14 yrs. This may be because children in the younger age group may not be managed within a TB clinic setting where reporting is more routine and often performed by the TB nurse. It is difficult to make comparisons between the $0-4$-yrs and 15 -yrs age groups due to the small number in the latter. In addition, even children seen in paediatric TB clinics may also have been preferentially notified to the established ETS system rather than the BPSU study.

In conclusion, this study shows that there is under-reporting, by $\sim 20 \%$, of childhood TB to ETS, especially of children aged $<5$ yrs. ETS provides a representative picture of the demographics of TB in children in the UK, but not the burden of disease. This is important as ETS is used to make strategic decisions about the provision of training and resources required for TB. It is vital that paediatricians are involved in the care of children with TB. Paediatricians should, however, work closely with local TB services and routinely report all cases of paediatric TB to ETS. Increased efforts should be made to ensure that all paediatric cases are reported to ETS in the future.

\section{SUPPORT STATEMENT}

S.S.S. Teo was partially funded by a UK Dept of Health research grant. The funding source had no involvement in study design; in the collection, analysis, or interpretation of data; in the writing of the report; or in the decision to submit the paper for publication.

\section{STATEMENT OF INTEREST}

None declared.

\section{ACKNOWLEDGEMENTS}

The authors would like to thank the British Paediatric Surveillance Unit Childhood Tuberculosis study group: D. Antoine (Health Protection Agency, London, UK), M. Cafferky (Temple Street Children's Hospital, Dublin, Ireland), P. Coen (Centre for Child Health, University of
London, London, UK), J. McMenamin (Health Protection Scotland, Glasgow, UK), W. Hoskyns (Leicester General Hospital, Leicester, UK); the British Paediatric Surveillance Unit (BPSU), supported by the Dept of Health, for facilitating the data collection; and the reporting clinicians, particularly those who completed the questionnaires. Any views expressed are those of the investigator and not necessarily those of the BPSU or the Dept of Health.

\section{REFERENCES}

1 www.hpa.org.uk/publications/PublicationDisplay. asp?PublicationID $=62$ Health Protection Agency Centre for Infections. Focus on Tuberculosis: Annual surveillance report 2006 - England, Wales and Northern Ireland. Last updated: November 2006. Last accessed: 12 March 2008.

2 www.hpa.org.uk/infections/topics_az/tb/pdf/MycobNet_10_Year_ Report.pdf Tuberculosis Section Communicable Disease Surveillance Centre Health Protection Agency London. The UK Mycobacterial surveillance network report 1994-2003, 10 years of MycobNet. Date last updated: December 2005. Date last accessed: 20 November 2006.

3 Pillaye J, Clarke A. An evaluation of completeness of tuberculosis notification in the United Kingdom. BMC Public Health 2003; 3: 31-35.

4 Shingadia D, El Bashir H, Aston A, et al. Paediatric tuberculosis in East London. Arch Dis Child 2002; 86: Suppl. 1, 65-70.

5 British Paediatric Surveillance Unit. 2004-5 Annual Report. Last updated: September 2005. Last accessed: 31 January 2008.

6 Fathoala B, Evans MR, Campbell IA, et al. Active surveillance for tuberculosis in Wales: 1996-2003. Arch Dis Child 2006; 91: 900-904.

7 Hook EB, Regal RR. Capture-recapture methods in epidemiology: methods and limitations. Epidemiol Rev 1995; 17: 243-264.

8 Knowles RL, Smith A, Lynn R, et al. Using multiple sources to improve and measure case ascertainment in surveillance studies: 20 years of the British Paediatric Surveillance Unit. J Public Health (Oxf) 2006; 28: 157-165.

9 Chemotherapy and management of tuberculosis in the United Kingdom, recommendations 1998. Joint Tuberculosis Committee of the British Thoracic Society. Thorax 1998; 53: 536-548.

10 National Collaborating Centre for Chronic Conditions. Tuberculosis: clinical diagnosis and management of tuberculosis, and measures for its prevention and control. London: Royal College of Physicians; 2006.

11 www.dh.gov.uk/assetRoot/04/11/43/68/04114368.pdf Department of Health. A Guide to Promote a National Service Framework for Children, Young People and Maternity Services. Shared Understanding of the Benefits of Managed Local Networks. 2005. Last updated: June 2005. Last accessed: 9 October 2006.

12 Anderson SR, Maguire H, Carless J. Tuberculosis in London: a decade and a half of no decline [corrected]. Thorax 2007; 62: 162-167. 